\title{
Das Leben ist Synchronisation. Möglichkeiten und Formen sozialer Teilhabe vor dem Hintergrund atypischer Arbeitszeiten
}

\author{
Bettina STADLER, Annika SCHÖNAUER, Anna ARLINGHAUS, Bernhard SAUPE, Hubert EICHMANN ${ }^{1}$ \\ Forschungs- und Beratungsstelle Arbeitswelt (FORBA), Wien
}

XIMES GmbH Wien

\begin{abstract}
Der Einfluss atypischer, d.h. langer oder außerhalb der üblichen Zeiten liegender Arbeitszeiten auf Gesundheit und Unfallrisiken ist gut belegt. Weniger umfassend dokumentiert ist der Zusammenhang zwischen atypischen Arbeitszeiten und den Möglichkeiten zu sozialer Teilhabe. In diesem Beitrag präsentieren wir Ergebnisse einer qualitativen Erhebung zu arbeitszeitbedingten Beeinträchtigungen sozialer Teilhabe in Österreich. Ziel der explorativ angelegten Untersuchung war, den Zusammenhang von langen Arbeitszeiten, Arbeit zu Randzeiten (Abend, Wochenende, Nacht) sowie eigenen Einflussmöglichkeiten und Planbarkeit der Arbeitszeit mit der sozialen Teilhabe der Beschäftigten zu untersuchen. Insbesondere atypische Lagen von Arbeitszeiten und fremdgesteuerte Flexibilität - im Sinne schlechter Planbarkeit der Arbeitsanforderungen - können die soziale Teilhabe negativ beeinflussen.
\end{abstract}

Schlüsselwörter: Arbeitszeit, atypische Arbeitszeiten, Beeinträchtigung, soziale Teilhabe, Freizeit

\section{Einleitung und Fragestellung}

In der alltäglichen Praxis der Arbeitszeitgestaltung verliert der gesetzliche Rahmen des AchtStunden-Tages schon seit einiger Zeit an Bedeutung und flexible Formen der Arbeitszeitgestaltung werden für immer mehr Beschäftigte zur gelebten Realität (Seifert 2005; Pomberger 2005; Kümmerling 2007; Astleithner/Stadler 2018). Auch durch die zum 1.9.2018 in Österreich in Kraft getretene Ausweitung der gesetzlichen Höchstgrenze der Arbeitszeit von täglich 10 auf 12

\footnotetext{
${ }^{1}$ Bettina Stadler, Annika Schönauer, Bernhard Saupe und Hubert Eichmann sind wissenschaftliche MitarbeiterInnen bei der Forschungs- und Beratungsstelle Arbeitswelt (FORBA), Wien: stadler@forba.at, schoenauer@forba.at, saupe@forba.at, eichmann@forba.at. Anna Arlinghaus ist Senior Consultant bei XIMES GmbH, Wien: arlinghaus@ximes.com.
}

DOI: http://dx.doi.org/10.18753/2297-8224-167 
Stunden und wöchentlich auf 60 Stunden wird die weitere Flexibilisierung von Arbeitszeiten ermöglicht.

Negative gesundheitliche Folgen langer Arbeitszeiten sind empirisch gut belegt (z.B. Amlinger-Chatterjee 2016). Längere Wochenarbeitszeiten hängen zudem grundsätzlich mit berichteter schlechterer Work-Life-Balance zusammen (Wirtz 2010), andererseits lassen sich auch positive Effekte sogenannter komprimierter Arbeitszeit (compressed working week) auf das Sozialleben finden (Tucker 2006; Arlinghaus et al. 2019). Insgesamt liegen jedoch zu arbeitszeitbedingter Beeinträchtigung der sozialen Teilhabe nur bedingt Erkenntnisse vor: Wöhrmann et al. (2020) führten kürzlich ein systematisches Review zu den Effekten von Schichtarbeit auf die Work-Life-Balance durch und Arlinghaus et al. (2019) beschrieben in ihrer Übersichtsarbeit Zusammenhänge zwischen verschiedenen Arbeitszeitmerkmalen auf das Sozialleben und die Familie. Kernergebnis beider Arbeiten ist, dass atypische Arbeitszeiten wie lange Arbeitszeiten, Nachtarbeit, irreguläre und schwer planbare Arbeitszeiten sowie Arbeit am Abend und am Wochenende mit Beeinträchtigungen des Sozial- und Familienlebens zusammenhängen. Eigener Einfluss der Beschäftigten auf ihre Arbeitszeitgestaltung wirkt dagegen positiv auf die wahrgenommene Work-Life-Balance und die Vereinbarkeit von Beruf und Privatleben (Arlinghaus et al. 2019; Wöhrmann et al. 2020).

Wie die o.g. AutorInnen anmerken, liegen jedoch unzureichende Befunde insbesondere zu den sozialen Effekten sehr langer täglicher und wöchentlicher Arbeitszeiten sowie dauerhafter Nachtarbeit vor, oder wurden bisher selten in Verbindung mit den sozialen Konstellationen der Beschäftigten untersucht. Darüber hinaus ist wenig darüber bekannt, wie die Beschäftigten ihre arbeitsfreie Zeit im Fall sozialer Beeinträchtigung oder bei Konflikten zwischen Arbeitszeit und sozialen Aktivitäten priorisieren.

Die Zielsetzungen dieses Beitrags beziehen sich daher auf die Bereitstellung hypothesengenerierender Erkenntnisse zur Frage, welche Beeinträchtigungen der sozialen Teilhabe für ArbeitnehmerInnen mit atypischen Arbeitszeiten einhergehen und welche Aspekte der sozialen Teilhabe im Fall von Beeinträchtigungen Vorrang haben. Dafür konzentrierten wir uns auf verschiedene Formen atypischer Arbeitszeiten, die in Betrieben unterschiedlicher Branchen untersucht wurden. Es handelt sich dabei um lange Arbeitszeiten (tägliche Arbeitszeiten über 8 Stunden oder wöchentlich über 40 Stunden) und eine von der Tagarbeit abweichende Arbeitszeitlage (z.B. früher Beginn, Spätdienst, Nachtdienst). Zusätzlich wurde der Aspekt der Planbarkeit von Arbeitszeit in den Scope einbezogen, da dieser in bisherigen Untersuchungen als wichtige Variable herausgearbeitet wurde (z.B. Arlinghaus et al. 2019).

\section{Soziale Teilhabe als Möglichkeit zu sozialer Interaktion}

Soziale Teilhabe wird häufig als gesellschaftliche, d.h. materielle, kulturelle und politische Partizipation verstanden, vor allem im Zusammenhang mit Chancengleichheit in der Integrationsforschung und Forschung zu Inklusion von gesellschaftlich benachteiligten Gruppen (z.B. Marschke/Brinkmann 2011). Der Begriff der sozialen Teilhabe an sich ist jedoch wenig trennscharf und verweist je nach Zielsetzung auf heterogene, wenngleich überlappende Deutungshorizonte. In unserem Verständnis gehen wir von Georg Simmels (2013 [1908]) Begriff der 
Vergesellschaftung aus. Er sieht den Gegenstand der Soziologie in sozialen Interaktionsprozessen, die er mit dem Überbegriff der Vergesellschaftung bezeichnet. Soziale Teilhabe sehen wir in diesem Sinne als eine Form der Vergesellschaftung. Auch Weber (2002 [1922]) und Tönnies (1920 [1887]) beschrieben Vergesellschaftung als einen Prozess, der aus Individuen Gesellschaftsmitglieder macht, indem er sie in unterschiedlicher Weise in den sozialen Zusammenhang integriert. Der Prozess, ein Gesellschaftsmitglied zu werden, vollzieht sich vor allem klassen-, ethnien- und geschlechtsspezifisch und unterliegt sich verändernden sozialhistorischen Bedingungen.

Wir verstehen soziale Teilhabe darauf aufbauend als soziale Interaktion mit relevanten Kontaktpersonen, zum Beispiel in Familie, Freundeskreis, Nachbarschaft und in Vereinen. Einen weiteren Aspekt bildet die soziale Interaktion in der Erwerbsarbeit via ArbeitskollegInnen und dem beruflichen Netzwerk (Hochschild 2006). Diese sind zwar in einer Gesamtbetrachtung ebenfalls von großer Bedeutung, der Fokus unserer Analyse liegt jedoch auf sozialer Teilhabe außerhalb der Erwerbsarbeit (siehe Tabelle 1).

Tabelle 1: Aspekte sozialer Teilhabe in der vorliegenden Studie

\begin{tabular}{|l|l|}
\hline Schwerpunkt in der Analyse & Exkludierte Aspekte \\
\hline Kernfamilie, PartnerIn, Kinder & Soziale Teilhabe in der Arbeit \\
\hline Familie, Verwandtschaft & Soziale Teilhabe online in der Arbeitszeit \\
\hline FreundInnen & \\
\hline Verein, Kurs, politische Aktivität etc. & \\
\hline
\end{tabular}

Quelle: eigene Darstellung

Mit unserem Beitrag untersuchen wir, ob und in welcher Form diese Aspekte sozialer Teilhabe durch atypische Arbeitszeiten negativ beeinflusst werden. Wir haben hierzu ein exploratives Vorgehen gewählt. Vorrangiges Ziel der Untersuchung ist es, wichtige Ansatzpunkte für zukünftige, auch quantitative Untersuchungen des Einflusses atypischer Arbeitszeiten auf soziale Teilhabe zu identifizieren. Daher wurden keine spezifischen Hypothesen untersucht.

\section{Methode}

Dieser Text basiert auf den Ergebnissen eines durch die Österreichische Forschungsförderungsgesellschaft (FFG) geförderten Projekts mit dem Titel Soziale Teilhabe als Herausforderung für Arbeitszeitgestaltung - Indikatoren-Entwicklung für faire Bewertungssysteme betrieblicher Gestaltung von Arbeitszeit, das zwischen Januar 2018 und Oktober 2019 in Österreich durchgeführt wurde (vgl. Eichmann 2019).

In den Jahren 2018/2019 wurden acht Betriebsfallstudien zu den Praktiken in Unternehmen im Umgang mit atypischen Arbeitszeiten und den Auswirkungen auf soziale Teilhabe durchgeführt. Die empirische Analyse konzentrierte sich auf vier unterschiedliche Branchen: stationäre und mobile (ambulante) Pflege, zweiteres wird in weiterer Folge ausschließlich als mobile 
Pflege bezeichnet, Sicherheitsdienstleistung, Hotellerie und dabei insbesondere Rezeption, sowie IT-Projektarbeit in einem internationalen Finanzdienstleistungsunternehmen und in zwei Start-Up-Betrieben. Diese Branchen wurden als idealtypische Beispiele ausgewählt, da dort jeweils wichtige atypische Arbeitszeitformen den Arbeitsalltag einer sehr großen Zahl von Beschäftigten prägen. Innerhalb dieser Branchen wurde jeweils mindestens ein Unternehmen ausgewählt und kontaktiert. Dabei wurde auf eine Verteilung der Unternehmen über ganz Österreich geachtet. Der Zugang zu den Unternehmen erfolgte über Kontakte zu BetriebsrätInnen bzw. über die Leitung der jeweiligen Organisation. Für jede Fallstudie wurden auf der Ebene der ausgewählten Abteilung bzw. der ausgewählten Berufsgruppen zwischen 4 und 10 Interviews mit Beschäftigten, VertreterInnen des Betriebsrates sowie mit Personen aus dem Management durchgeführt (insgesamt 36 Personen). Auf dieser Basis können vertiefende Erkenntnisse zu den Wirkungen von Arbeitszeiten auf soziale Teilhabe gewonnen werden, die Ergebnisse sind jedoch nicht repräsentativ in einem statistischen Sinne.

Die Stichprobe setzte sich wie folgt zusammen:

- Stationäre und mobile Pflege: 18 Personen aus 2 Unternehmen

- Sicherheitsdienstleistung: 4 Personen aus 1 Unternehmen

- Hotellerie/Rezeption: 4 Personen aus 2 Unternehmen

- IT-Projektarbeit: 10 Personen aus 3 Unternehmen (Finanzdienstleistung und IT-StartUps)

Bei der Auswahl der InterviewparterInnen wurde auf eine breite, dem jeweiligen Setting entsprechende Streuung hinsichtlich des Geschlechts, der Altersgruppe inkl. Betreuungspflichten für Kinder oder sonstige Personen, der Formalbildung/Beruf/Position und der Beschäftigungsdauer geachtet. Ziel war, die Struktur der Beschäftigten mit den jeweils vorhandenen Variationen möglichst gut abzubilden.

Für die empirische Analyse der Praktiken und der Gestaltbarkeit atypischer Arbeitszeiten und deren Auswirkungen auf soziale Teilhabe wurde eine breite Definition des Begriffs atypische Arbeitszeiten verwendet. Damit sind alle Formen von Arbeitszeiten angesprochen, die außerhalb der traditionellen Arbeitszeiten, d.h. an Wochentagen und tagsüber, geleistet werden. Die Formen atypischer Arbeitszeiten umfassen damit folgende Merkmale:

- Dauer der Arbeitszeit im Sinne vor Arbeitszeiten über 8 Stunden pro Tag

- Lage der Arbeitszeit im Sinne von Arbeit am frühen Morgen, spät am Abend, in der Nacht oder am Wochenende

- Planbarkeit der Arbeitszeiten vor allem in Form von kurzfristigen Änderungen von Dienstplänen und Arbeitseinsätzen mit kurzer oder nicht vorhandener Ankündigungsfrist. Mit dem Planungshorizont der Arbeitszeit ist gemeint, wie lange im Voraus die Beschäftigten üblicherweise wissen, ob und wann sie an einem bestimmten Tag arbeiten, bzw. inwieweit es zu fremdbestimmten kurzfristigen Änderungen der geplanten Arbeitszeit kommt. 
In allen Betriebsfallstudien wurden leitfadengestützte Interviews in Form von Einzel- oder Gruppeninterviews durchgeführt. Für alle drei untersuchten Gruppen (Beschäftigte, BetriebsrätInnen und VertreterInnen des Managements) wurden eigene Leitfäden formuliert. Ausgehend von einer Arbeitsdefinition von sozialer Teilhabe wurde in den Interviews nach der persönlichen Lebenssituation, der vertraglichen Arbeit und der gelebten Praxis der Arbeitszeitgestaltung gefragt. Im Detail wurde anschließend nach von den Befragten wahrgenommenen Wirkungen ihrer Arbeitszeit auf ihre soziale Teilhabe in einer Vielzahl von Aspekten ihres Lebens (Familienleben, Freizeit, Kultur, Sport, Erholung, Gesundheit etc.) gefragt. Die Interviews wurden vollständig transkribiert, anonymisiert und nach der Methode der soziologischen Inhaltsanalyse (Mayring/Fenzl 2019) ausgewertet. Die Ergebnisse wurden in Form von Betriebsfallstudien aufbereitet.

\section{Datengrundlage}

In den unterschiedlichen Branchen wurden verschiedene Kombinationen der untersuchten Arbeitszeitmodelle vorgefunden. Tabelle 2 zeigt einen Überblick über die ausgewählten Branchen, die Anzahl der Interviews und die praktizierten Arbeitszeitmodelle. Im folgenden Kapitel sind die Ergebnisse entlang der unterschiedlichen Arbeitszeitphänomene aufbereitet. Die untersuchten Branchen werden beispielhaft präsentiert. Folglich finden sich in diesem Text nicht alle Brachen im gleichen Ausmaß wieder.

Tabelle 2: Überblick untersuchter Branchen und Arbeitszeitphänomene

\begin{tabular}{|l|c|c|c|c|c|}
\hline $\begin{array}{l}\text { Untersuchte Arbeitszeit- } \\
\text { phänomene }\end{array}$ & $\begin{array}{c}\text { Mobile } \\
\text { (ambulante) } \\
\text { Pflege }\end{array}$ & $\begin{array}{c}\text { Stationäre } \\
\text { Pflege }\end{array}$ & $\begin{array}{c}\text { Sicherheits- } \\
\text { dienstleis- } \\
\text { tung }\end{array}$ & $\begin{array}{c}\text { Hotellerie / } \\
\text { Rezeption }\end{array}$ & $\begin{array}{c}\text { IT-Projekt- } \\
\text { arbeit }\end{array}$ \\
\hline Lange Arbeitszeiten & $\mathrm{X}$ & $\mathrm{X}$ & $\mathrm{X}$ & & $\mathrm{X}$ \\
\hline $\begin{array}{l}\text { Arbeit am Abend / frühen } \\
\text { Morgen }\end{array}$ & $\mathrm{X}$ & $\mathrm{X}$ & $\mathrm{X}$ & $\mathrm{X}$ & $\mathrm{X}$ \\
\hline Nachtarbeit & $\mathrm{X}$ & $\mathrm{X}$ & $\mathrm{X}$ & $\mathrm{X}$ & \\
\hline Wochenendarbeit & $\mathrm{X}$ & $\mathrm{X}$ & $\mathrm{X}$ & $\mathrm{X}$ & \\
\hline
\end{tabular}




\begin{tabular}{|c|c|c|c|c|c|}
\hline & $\begin{array}{l}\text { Mobile } \\
\text { (ambulante) } \\
\text { Pflege }\end{array}$ & $\begin{array}{l}\text { Stationäre } \\
\text { Pflege }\end{array}$ & $\begin{array}{l}\text { Sicherheits- } \\
\text { dienstleis- } \\
\text { tung }\end{array}$ & $\begin{array}{l}\text { Hotellerie / } \\
\text { Rezeption }\end{array}$ & $\begin{array}{l}\text { IT-Projekt- } \\
\text { arbeit }\end{array}$ \\
\hline \multirow{3}{*}{ InterviewpartnerInnen } & $\begin{array}{l}\text { Pflege(fach) } \\
\text { assistentInnen } \\
\text { ( } 3 \text { männl., } 1 \\
\text { weibl.) }\end{array}$ & $\begin{array}{l}\text { Pflegefach- } \\
\text { kräfte ( } 8 \\
\text { weibl.) }\end{array}$ & \multirow{3}{*}{$\begin{array}{l}\text { Sicherheits- } \\
\text { personal (4 } \\
\text { männl.) }\end{array}$} & $\begin{array}{l}\text { Rezeptionis- } \\
\text { ten }(2 \\
\text { männl.) }\end{array}$ & $\begin{array}{l}\text { Mitarbeiter } \\
\text { Innen Fi- } \\
\text { nanzdienst- } \\
\text { leistung (5 } \\
\text { männl., 1 } \\
\text { weibl.) }\end{array}$ \\
\hline & $\begin{array}{l}\text { Diplomierte } \\
\text { Pflegekräfte (1 } \\
\text { männl., } 2 \\
\text { weibl.) }\end{array}$ & $\begin{array}{l}\text { Einrich- } \\
\text { tungsleitung } \\
\text { (1 weibl.) }\end{array}$ & & $\begin{array}{l}\text { Betriebsrat } \\
\text { (1 männl.) }\end{array}$ & $\begin{array}{l}\text { Mitarbeiter } \\
\text { Innen Start- } \\
\text { Ups (3 } \\
\text { männl., 1 } \\
\text { weibl.) }\end{array}$ \\
\hline & $\begin{array}{l}\text { Verwaltung } \\
\text { Management } \\
\text { (weibl.) }\end{array}$ & & & $\begin{array}{l}\text { Geschäfts- } \\
\text { führung (1 } \\
\text { männl.) }\end{array}$ & \\
\hline
\end{tabular}

Quelle: eigene Darstellung

\section{Mobile Pflege}

Das Fallstudienunternehmen aus der mobilen Pflege ist ein Anbieter für Pflege- und Betreuungsdienstleistungen. Teil der Fallstudie sind diplomierte Gesundheits- und Krankenpflegekräfte, PflegefachassistentInnen und PflegeassistentInnen. Die befragten PflegeassistentInnen und PflegefachassistentInnen arbeiteten in einem langfristig planbaren 2-Schichtmodell mit 4bis 9-stündigen Diensten. Diplomierte Pflegekräfte arbeiten ebenfalls in einem 2-Schichtmodell in Gleitzeit von Montag bis Freitag mit Wochenenddiensten.

\section{Stationäre Pflege}

Die untersuchte Einrichtung ist ein Pflegeheim in kommunaler Trägerschaft mit zwei in räumlicher Nähe zueinander gelegenen Standorten. Das betriebliche Arbeitszeitmodell ist ein in Pflege- und Behinderteneinrichtungen allgemein übliches Turnusmodell. Einem Nachtdienst (ohne Schlafmöglichkeit) stehen unterschiedlich lange Tagdienste gegenüber.

\section{Sicherheitsdienstleistung}

Die Interviews für die hier präsentierte Studie wurden in einer Niederlassung eines großen Sicherheitsunternehmens durchgeführt. Drei der vier Interviewpartner leisteten zum Zeitpunkt des Interviews oder in einer früheren Funktion Nachtdienste. So wie die Mehrzahl der Beschäftigten im Bewachungsgewerbe verfügen die Befragten über geringe formale Qualifikationen. Die Wochenarbeitszeit von Wachorganen im Bewachungsgewerbe ist im Kollektivvertrag grundsätzlich mit 8 Stunden pro Tag bzw. 40 Stunden pro Woche festgelegt. Bei Arbeitsbereitschaft kann die Wochenarbeitszeit auf 48 Stunden pro Woche bzw. 12 Stunden pro Tag ausgeweitet werden. Innerhalb einer Woche kann die Arbeitszeit unter bestimmten Umständen ohne Zuschläge sogar auf bis zu 60 Stunden ausgedehnt werden. Verglichen mit anderen Branchen 
ist das Lohnniveau im Bewachungsgewerbe sehr niedrig, auch die Zuschläge für Nachtarbeit sind sehr gering.

Hotellerie (Rezeption)

Die Interviews im Bereich der Hotellerie wurden in zwei verschiedenen Hotels in einer Großstadt in Österreich durchgeführt. Zwei der Befragten leisten regelmäßig Nachtdienste an der Rezeption. Für die Nachtdienste sind in den Unternehmen eigene MitarbeiterInnen zuständig, die keine anderen Dienste übernehmen, es handelt sich also um Arbeitsplätze mit Dauernachtarbeit.

Projektarbeit bei einem internationalen Finanzdienstleistungsunternehmen

Der analysierte Fallbetrieb ist die rechtlich selbstständige IT-Tochtergesellschaft eines international tätigen Finanzdienstleistungsunternehmens mit Sitz in einer großen Stadt in Österreich. In Bezug auf Formalqualifikationen hat die große Mehrheit der Beschäftigten in der IT-Tochter entweder eine höhere technische Ausbildung (v.a. Informatik) bzw. teilweise ein Studium absolviert. Für diese Fallstudie wurden in der Zentrale Interviews mit Personen aus unterschiedlichen Hierarchiestufen durchgeführt. Selten werden dort mehr als 10-Stunden-Dienste geleistet, weder das Unternehmen noch die MitarbeiterInnen zeigen Interesse an längeren Arbeitszeiten. Fallweise notwendige Wochenenddienste, z.B. wegen Softwareumstellungen, sind lange vorher geplant und treten nur etwa einmal im Quartal auf. Im Unternehmen gibt es ein Angebot für Home-Office, nur ein Teil der interviewten MitarbeiterInnen nimmt dieses jedoch auch in Anspruch.

\section{Startup-Betriebe in der Internet-Branche}

Die beiden untersuchten Startup-Unternehmen der Internet-Branche sind zum Zeitpunkt der Interviewdurchführung in der ersten Hälfte 2019 jünger als zwei Jahre und befinden sich dementsprechend in einer dynamischen bis chaotischen Aufbauphase. Startup A betreibt eine Online-Matchingplattform und listet auf der eigenen Website ein Team von mehr als 15 Personen auf. Nach Auskunft der Gesprächspartner aus Startup B, die beide über Dienstverträge beschäftigt sind, arbeiten am Bürostandort Wien weniger als zehn Personen in einem Angestelltenverhältnis. Formal wurde auch in diesen Unternehmen zwischen ArbeitgeberIn und ArbeitnehmerInnen eine bestimmte Anzahl von Arbeitsstunden vereinbart. In der Praxis ist aber für beide Seiten klar, dass die Arbeitszeitrealität von diesen Stunden häufig abweicht. Immer wieder kommt es zu längeren Arbeitszeiten und auch die Grenzen zwischen Arbeitsleistung und privaten Tätigkeiten verschwimmen.

\section{Ergebnisse: Beeinträchtigung sozialer Teilhabe bei atypischen Arbeitszeiten}

\section{Dauer der Arbeitszeit}

Wie oben bereits kurz beschrieben, werden in mehreren untersuchten Unternehmen regelmäßig lange Arbeitszeiten geleistet. Dies geschieht sowohl in Form von langen täglichen Diensten als auch in Form von langen Wochenarbeitszeiten. Ob es sich bei den langen Arbeitszeiten um 
Überstunden oder Mehrarbeitsstunden handelt, wird nicht systematisch unterschieden. Der Grund dafür ist, dass aufgrund von Durchrechnungszeiträumen zum Zeitpunkt der Leistung von Arbeit oft gar nicht bekannt ist, ob es sich um Mehr- oder Überstunden handelt. Im Folgenden wird dargelegt, wie die Beschäftigten in den hier untersuchten Organisationen ${ }^{2}$ lange Arbeitszeiten erleben und welche Vor- und Nachteile sie damit verbinden.

\section{Dauer der Arbeitszeit: Pflegeheim}

Lange Dienste treten im Pflegeheim zum einen in Gestalt der Nachtdienste auf, die grundsätzlich zwölf Stunden dauern. Auch die durchgehenden Tagdienste dauern laut Beschreibung der Beschäftigten in der Praxis gelegentlich bis zu zwölf Stunden. In den Schilderungen wird deutlich, dass es Unterschiede zwischen gelebter Praxis und Arbeitszeitregelung gibt. So wird von einer Mitarbeiterin hervorgehoben, dass die offizielle Dauer 11 Stunden und 30 Minuten beträgt. Eine Vorgesetzte spricht wiederum von einer Höchstarbeitsgrenze für Tagdienste von elf Stunden.

Was die Bewertung der langen Dienste betrifft, heben die befragten Mitarbeiterinnen häufig die damit verbundenen Vorteile hervor. Neben der Bezahlung (Zuschlag für Nachtarbeit) ist dies insbesondere der Umstand, dass lange Dienste mit langen Freizeitblöcken einhergehen:

Und das ist halt auch als junger Mensch ganz fein, weil du kannst dann einfach einmal weg und verreisen, ohne dass du Urlaub eintragen musst. [...] Ich bin eher ein Freund von 12-Stunden-Diensten und Durch-Diensten. Aber, vermutlich, wenn ich selber einmal Kinder haben werde, dann wäre wahrscheinlich auch die familienfreundlichere Variante mit geteilten Diensten auch eine Option. Aber ich bin eher der Meinung, ich komme lieber für mehr Stunden herein und weniger Tage und dann ist es vorbei, als wie ich komme fünfmal oder teilweise sechsmal in der Woche rein und hackle [Anm.: arbeite] halt 5 Stunden. Das bringt für mich nicht wirklich viel. Genau. (Pflegeheim MA 3)

Lange Dienste werden, wenn sie in eine entsprechende Organisation der Arbeitszeit mit darauffolgenden langen Freizeitblöcken eingebunden sind, von vielen Arbeitnehmerinnen des untersuchten Pflegeheims durchaus geschätzt. Um jedoch Belastungen unter anderem durch lange Dienste, aber auch durch Flexibilitätsanforderungen ausgleichen zu können, arbeiten viele Beschäftigte im Pflegebereich in Teilzeit.

\section{Dauer der Arbeitszeit: Sicherheitsdienst}

In diesem Unternehmen zeigt sich, dass die Beschäftigten neben ihren Belastungen durch lange Arbeitszeiten insbesondere die positiven Aspekte im Zusammenhang mit dem Einkommen schildern. Beispielsweise arbeitet ein interviewter Mitarbeiter entsprechend seines Dienstplans von 19 Uhr abends bis 5 Uhr in der Früh, diese Arbeitszeit von 10 Stunden pro Tag wird in folgender Weise positiv bewertet:

\footnotetext{
${ }^{2}$ Im untersuchten Hotel gibt es tägliche Arbeitszeiten von 8,5 Stunden, die im Bereich der Nachtdienste auch weitgehend eingehalten werden. Aus diesem Grund wird in diesem Abschnitt nicht näher auf die Wirkungen langer Arbeitszeiten in der Hotellerie eingegangen.
} 
Und bei uns ist es z. B. so: 8 Stunden ist für jeden Mitarbeiter für uns nicht passend. Ja, das ist wirklich so. Weil bei uns ist auch - das muss man ehrlich dazusagen natürlich auch lohnmäßig. Und viele arbeiten auch Überstunden, die sagen natürlich, mit den 10 Stunden ist es für sie akzeptabel, dass sie 4 Tage arbeiten, normal, und einmal einen 5. Tag machen, und somit haben sie trotzdem noch 2 Tage frei. (Sicherheit MA 1)

Ein anderer Mitarbeiter hat 12-Stunden-Dienste vereinbart, seine Arbeitszeit beschreibt er folgendermaßen:

Das heißt, viermal in der Woche muss ich arbeiten laut meinem Vertrag. Es kann sein, dass ich 48 bis 60 Stunden mache. Ich habe auch 72 Stunden auch einmal gehabt. (Sicherheit MA 3)

Als besonders problematisch - u.a. für ihre soziale Teilhabe - beschreiben Mitarbeiter im Sicherheitsdienst, wenn lange Dienste während der Nacht zum Beispiel durch plötzlich eintretende Notfälle oder technische Probleme länger dauern als geplant. Ein oder zwei zusätzliche Arbeitsstunden führen dann dazu, dass die Schlafphase deutlich später beginnt, kürzer ausfällt oder insgesamt schwerer möglich ist. Dies beeinflusst anschließend das soziale Leben am folgenden Tag negativ.

Wichtig für die Gestaltung der Arbeitszeit ist es hier also, einen Weg zwischen der finanziellen Notwendigkeit, viele Stunden zu leisten, um einen höheren Lohn zu erhalten und der Bewältigung der Dauer und Lage der Dienste zu finden.

\section{Dauer der Arbeitszeit: IT-Projektarbeit}

Im untersuchten IT-Tochterunternehmen berichten Interviewte über eine Ausrichtung auf das klassische Normalarbeitszeitmodell mit wenig Mehrstunden. In den mittleren Berufsjahren mit +/- 40 Jahren und vor dem Hintergrund eines ohnehin soliden Gehalts dominiert die Einschätzung, nicht nur der Erwerbsarbeit, sondern auch der Freizeit ausreichend Raum zu geben. Ein 42-jähriger Informatiker skizziert seine Situation wie folgt:

Wenn ich jeden Tag zehn Stunden arbeiten wollte, dann könnte ich das auch. Ich habe genug Arbeit da. Ich mache keine Minusstunden, geht auch nicht als Angestellter, ich mache auch nicht viel Überstunden, aber es ist definitiv genug Arbeit da, um viele Überstunden zu machen. (...) Ich habe mir früher eigentlich jedes Monat Überstunden auszahlen lassen und länger gearbeitet, eben um mehr zu verdienen. Und, irgendwann ist es ja nicht mehr so wichtig gewesen, mehr zu verdienen. (...). Hätte ich eine Führungsposition, wäre die Anforderung ganz klar zu Überstunden. (ITTochter MA 4)

Hier sehen wir ein gut planbares Regel-Arbeitszeitmodell. Soziale Teilhabe ist aufgrund der gut planbaren Arbeitszeiten zu sozial üblichen Zeiten möglich. 


\section{Dauer der Arbeitszeit: Start-Ups}

Trotz langer Arbeitszeiten, stellt sich die Situation im Bereich eines Start-Ups gänzlich anders dar. Die Grenzen zwischen Arbeit und Freizeit sind vergleichsweise wenig klar abgesteckt. Lange Arbeitstage sind Teil der als selbstverständlich betrachteten Firmenkultur. Nach dem regulären Arbeitstag bildet sich eine interviewte Person beispielsweise freiwillig am Arbeitsplatz weiter. Begründet wird dies mit Interesse an der Arbeit und der Tatsache, dass man ja selbst auch davon profitieren würde. Wichtig ist hier aber nicht zu vergessen, dass die intensive Gründungsphase eines Start-Ups normalerweise als vorübergehend angesehen wird. Auch Einschränkungen in der außerberuflichen sozialen Teilhabe werden vor diesem Hintergrund leichter in Kauf genommen. Im besten Fall überwindet das Unternehmen diese intensive Phase und gelangt nach einiger Zeit in ruhigeres Fahrwasser mit geregelt(er)en Arbeitszeiten. Ein Mitarbeiter schildert die aktuellen Praktiken folgendermaßen:

Ich glaube, es sind 40 Stunden. Überstunden werden bezahlt, wenn ich ausdrücklich darum gebeten wurde, diese zu verrichten. Also wenn ich nicht gebeten wurde, muss ich sie nicht machen und sie werden auch nicht vergütet. Das ist der Vertrag. (...) Von den Arbeitsstunden, ich schreibe sie mir auf, aber das ist eher so für mich. (Startup A MA 1)

Von diesem Mitarbeiter sind keine Unmutsäußerungen vernehmbar, obwohl er häufig mehr Stunden verrichtet, als im Arbeitsvertrag vorgesehen. Es sei der unausgesprochene Deal mit diesem Arbeitgeber, dass er im Gegenzug für die Chance zu einem Neustart als Programmierer bzw. für den Weiterbildungskurs, für den er oft die Büroräumlichkeiten bis spät in die Nacht nutzt, bei Bedarf Mehrarbeit leistet.

\section{Zusammenschau: Dauer der Arbeitszeit}

Lange, über 8 Stunden hinausgehende Arbeitszeiten sind tendenziell sozial belastend für die ArbeitnehmerInnen aber zumindest aus einer kurzfristigen Perspektive auch mit Vorteilen verbunden. Ein Motiv für lange Arbeitszeiten sind neben höherem Einkommen oder einer Investition in die Karriere oder den Erfolg des Unternehmens im Falle der Start-Ups häufig die langen Freizeitphasen. Geht die Grenze zwischen bezahlter Arbeit und Freizeit beispielsweise durch regelmäßige, unbezahlte Vorbereitungsarbeit verloren (wie es in der mobilen Pflege vorkommt), wird dies sehr negativ bewertet.

Einen Überblick - basierend auf einer vergleichenden branchenübergreifenden Analyse zum Einfluss der verschiedenen Faktoren im Zusammenhang mit der Dauer von Arbeitszeit und sozialer Teilhabe gibt Tabelle 3. 
Tabelle 3: Einfluss der Dauer der Arbeitszeit auf soziale Teilhabe

\begin{tabular}{|l|c|c|}
\hline Dauer der Arbeitszeit & $\begin{array}{c}\text { Belastend für soziale } \\
\text { Teilhabe }\end{array}$ & Hilfreich für soziale Teilhabe \\
\hline Große Häufigkeit langer Dienste & $\bullet$ & \\
\hline Lange Freizeitphasen & & $\bullet$ \\
\hline $\begin{array}{l}\text { Zeitbonus für belastende Ar- } \\
\text { beitszeiten }\end{array}$ & & $\bullet$ \\
\hline Reduzierte Arbeitsstunden ${ }^{1)}$ & & \\
\hline $\begin{array}{l}\text { Keine / zu kurze Erholungspha- } \\
\text { sen zwischen den Diensten }\end{array}$ & $\bullet$ & \\
\hline
\end{tabular}

1) Häufig auch als Coping-Strategie, ist zugleich verbunden mit niedrigem Einkommen.

Quelle: Eigene Darstellung

\section{Lage der Arbeitszeit}

Neben langen Arbeitszeiten werden vor allem auch Arbeitszeiten am Abend, am frühen Morgen, in der Nacht oder am Wochenende zu den atypischen Arbeitszeiten gerechnet. In den nächsten Abschnitten werden wieder die Befunde aus den Branchen mit regelmäßig vorkommenden atypischen Arbeitszeitlagen dargestellt und mit Zitaten aus den Interviews illustriert.

\section{Lage der Arbeitszeit: Mobile Pflege}

Spätdienste enden in der untersuchten Organisation üblicherweise um 19:00 oder 20:00. Folgt darauf ein Frühdienst, so beginnt dieser am nächsten Tag meist um 6:00. Obwohl die gesetzlichen Ruhezeiten eingehalten werden, zeigt sich, dass die Abendfreizeit für die Beschäftigten nur sehr begrenzt nutzbar ist, was von vielen Beschäftigten als große Einschränkung erlebt wird:

Weil man trifft seine Freunde am Abend, am Wochenende, am Freitag oder wie auch immer, oder Stammtische sind auch teilweise unter der Woche. Aber es ist halt dann, man muss halt dann sagen: Okay, ich kann nicht kommen, weil ich habe am nächsten Tag, am nächsten Tag muss ich früher arbeiten, und am Tag davor habe ich eben Spätdienst. Und dann geht es halt nicht, außer ich bin halt dann halbtot in der Arbeit, aber das will ich den Leuten nicht zumuten und mir selber auch nicht. (mobile Pflege $P A$ 1)

Eine weitere zentrale Einschränkung für die Nutzbarkeit der Freizeit besteht darüber hinaus durch die stark variierende Lage der freien Tage. Das betrifft einerseits die Wochenenden aber auch die Wochentage. Die Beschäftigten beklagen, dass regelmäßige Termine, die auf einen fixen Wochentag fallen, nicht wahrgenommen werden können:

Ich würde gerne unterm Wochenende einmal einen Tanzkurs starten, weil ich das gerne mache. Im Moment über die Winterzeit in dem Job nicht wirklich möglich, weil 
es dann oft heißt: Der Kollege ist krank, kannst du einspringen? Ah, ja, nicht wirklich umsetzbar in der Branche. Nein. Und, wie gesagt, am Wochenende die ganzen, die haben alle frei, die wollen natürlich wissen: Was machst du nächstes Wochenende? Gehen wir fort? Oder einfach: „Kommst du?" „Ich kann nicht, ich habe Spätdienst. Ich kann nicht, ich habe Frühdienst. Ich weiß nicht, wann ich heimkomme. Also es ist dann schon teilweise sehr, sehr schwierig, das alles unter einen Hut zu bringen. (mobile Pflege DGKP 3)

In den Interviews mit den Beschäftigten finden sich Hinweise, dass die atypischen Dienstzeiten mitunter auch als körperlich belastend erlebt werden und Freizeitaktivitäten dadurch beeinträchtigt werden:

Ja, also den letzten Nachtdienst habe ich am Freitag gehabt jetzt zufällig, aber da haben meine Kinder Schikurs gehabt, und da habe ich am Samstag mitgehen müssen. (lacht) Und das war dann schon zäh, also das merkt man brutal. Also wenn was zum Tun ist, geht das dann eh am Tag, aber so, ja (...). Also hinsetzen darf man sich da nicht, also weil man einfach wirklich kaputt ist. Ja. Und das merkt man schon ein paar Tage danach immer, also ich merke es noch. (Pflegeheim MA 4)

\section{Lage der Arbeitszeit: Sicherheitsdienste}

Die Beschäftigten aus der Sicherheitsbranche stehen auch in finanzieller Hinsicht unter besonderem Druck, lange und flexible Arbeitszeiten anzunehmen. Der generelle Befund der Gesprächspartner: an Tagen mit Nachtarbeit bleibt nur schwer Zeit für die Pflege eines Soziallebens:

Duschen, essen, Kinder, Schlafengehen. (lacht) Also das sind die... Man versucht dann trotzdem, mit der Frau vielleicht irgendwas zu unternehmen, kurzfristig. Ja, es geht sich auch zweitweise aus. Man ist halt sehr, sehr müde, aber es geht. (Sicherheit MA 3)

Hinzu kommt, dass nicht nur während der Woche gearbeitet wird, sondern Dienste auch am Wochenende geleistet werden müssen. Vor allem diese Wochenenddienste werden als sehr einschränkend für das Sozialleben beschrieben. Kurzfristig können die sozialen Kontakte aufrechterhalten werden. Wird diese Form der Arbeitszeit aber über einen längeren Zeitraum beibehalten, gehen Freundschaften unweigerlich verloren. Berufliche Beziehungen können dies zum Teil kompensieren, ansonsten reduzieren sich die Kontakte sukzessive auf den familiären Bereich:

Der Freundeskreis bezieht sich dann, sagen wir, auf Kollegen zum Teil, und bezieht sich dann eigentlich, so Freundeskreis, auf die Familie netto mehr, so in die Richtung, weil so viele, die genau in der Zeit. ... sagen wir einmal, die 12-Stunden-Dienste haben, so viele gibt es ja nicht in die Richtung. (Sicherheit BR 1) 


\section{Lage der Arbeitszeit: Hotellerie}

Die Nachtportiere im untersuchten großen Stadthotel haben fix eingeteilte Nachtdienste. Plötzlich auftretende Personalknappheit z.B. durch Krankenstände wird durch zusätzliches Personal abgedeckt. Wohl vor allem auch wegen der guten Planbarkeit berichten die Gesprächspartner, dass dieses Arbeitszeitmodell Vorteile für die Vereinbarkeit ihrer Arbeit und Familienleben hat. Mehrere Gesprächspartner schätzen an ihrer Arbeit, dass sie tagsüber zu Hause sind und sich, nachdem sie ihren Schlaf nachgeholt haben, am Nachmittag um ihre Kinder kümmern können.

Zugleich ist große Disziplin bei den Schlafenszeiten erforderlich, dies betrifft nicht nur die ArbeitnehmerInnen selbst sondern das ganze familiäre Umfeld. Nur so kann dafür gesorgt werden, dass es gelingt, auch zu atypischen Zeiten ausreichend Schlaf zu finden:

Weil, dass wir in der Nacht arbeiten, das heißt nicht, dass wir auch eine Disziplin nicht haben müssen. Also es muss genau fixiert sein, wann wir ins Bett gehen. (Hotel MA 1)

Eine Verlagerung des Schlafes auf atypische Zeiten ist aber trotz guter Planung und Routine mit Belastungen verbunden. So berichten Gesprächspartner von tagsüber immer wieder auftauchender Müdigkeit.

Belastend sind darüber hinaus besonders die Arbeitszeiten am Wochenende: „Die Kinder fühlen sich irgendwie benachteiligt in diese Richtung, weil man kann nicht alle Aktivitäten immer auf die 2. oder 3. Woche verschieben" (Hotel MA 2). Aber auch am allgemeinen sozialen Leben nehmen die Gesprächspartner, wenn sie nachts arbeiten, weniger teil: „Und, ja, sozial, natürlich, wir leben wie Vampire. Also in der Nacht aktiv, am Tag sind wir im Bett“ (Hotel MA 1). Dies zeigt sich beispielsweise anhand von alltäglichen Ritualen wie dem Frühstück: „Sagen Sie mir, was ist Frühstück für mich? (...) Ist Frühstück jetzt in der Früh, oder? Für mich ist es das Abendessen vielleicht oder wenn ich ins Bett gehe" (Hotel MA 1).

Die zwar geplanten, aber doch wechselnden Nachtdienste führen somit klar zu Einschränkungen im Sozialleben. Ein Gesprächspartner berichtet, dass es schwierig ist, Freunde zu treffen bzw. diese sich seltener melden, da für sie nicht absehbar ist wann er arbeitet und wann er frei hat. Für die während der Nacht arbeitenden Portiere geht der Gewinn an sozialer Zeit für die Familie also auf Kosten der Zeit mit Freunden.

\section{Zusammenschau Lage der Arbeitszeit}

Unterschiedliche Arbeitszeitlagen haben unterschiedliche Auswirkungen auf die soziale Teilhabe der ArbeitnehmerInnen. Es lassen sich jedoch einige allgemeine Zusammenhänge aus den Interviews ableiten:

- Frühe Beginnzeiten (mobile und stationäre Pflege) schränken bereits die Gestaltung des Vorabends ein. Wer sehr früh am Morgen zur Arbeit eingeteilt ist, vermeidet es meist, soziale Aktivitäten für den Vorabend zu planen.

- Späte Dienste sind ebenso mit negativen Auswirkungen verbunden wie etwa der Einschränkung sozialer Aktivitäten am Abend.

- Bei Nachtarbeit muss zwischen gelegentlicher und Dauernachtarbeit unterschieden werden. 
Bei gelegentlicher Nachtarbeit (z.B. in der stationären Pflege) laufen soziale Aktivitäten häufig ähnlich den Phasen ohne Nachtarbeit weiter. Es bleibt dann zu wenig Zeit für Erholung. Menschen, gerade jene mit Kindern, nehmen wenig Rücksicht auf ihre eigenen Erholungsbedürfnisse. Bei Dauernachtarbeit besteht während der Arbeitsphasen keine Zeit für soziale Teilhabe außerhalb der Kernfamilie. Das gesamte Leben (zum Teil auch der Familie) ist auf Nachtarbeit ausgerichtet. Schlafstrategien erfordern Disziplin von dem/der ArbeitnehmerIn und dem Umfeld. NachtarbeiterInnen müssen Zeiten der Erholung genau planen und einen sehr disziplinierten Lebensstil pflegen. Wird die Planung durch kurzfriste Verlängerung des Dienstes gestört, ist die Erholung nach der Arbeit, aber auch die Teilnahme am Familienleben gefährdet. Als Vorteil wird Zeit für Kinderbetreuung erwähnt, die aufgrund der Nachtarbeit tagsüber vorhanden ist. Da Treffen mit Freunden und Familie, sowie andere Anlässe wie Konzerte, üblicherweise am Wochenende stattfinden, sind Beschäftigte mit Nachtdienst dann bei diesen Ereignissen immer wieder nicht dabei.

An die Grenze des physisch und sozial Leistbaren gelangen ArbeitnehmerInnen, wenn Nachtdienste mit Tagdiensten wechseln und zusätzlich kurzfristig Dienste eingeteilt werden. Aus den Sicherheitsdiensten werden solche Arbeitssituationen berichtet und zugleich beschrieben, dass dies ein Grund für die hohe Fluktuation in der Branche ist.

Einen Überblick - basierend auf einer vergleichenden branchenübergreifenden Analyse zum Einfluss der verschiedenen Faktoren im Zusammenhang mit der Lage von Arbeitszeit und sozialer Teilhabe gibt Tabelle 4 .

Tabelle 4: Einfluss des Faktors atypische Lage der Arbeitszeit auf soziale Teilhabe

\begin{tabular}{|l|c|c|}
\hline \multicolumn{1}{|c|}{ Lage der Arbeitszeit } & $\begin{array}{c}\text { Belastend für soziale Teil- } \\
\text { habe }\end{array}$ & $\begin{array}{c}\text { Hilfreich für soziale } \\
\text { Teilhabe }\end{array}$ \\
\hline Frühe Beginnzeiten & $\bullet$ & \\
\hline Späte Dienste & $\bullet$ & \\
\hline Wechselnde Randdienste & $\bullet$ & \\
\hline Nachtdienste & $\bullet$ & \\
\hline $\begin{array}{l}\text { Nachtdienste in Kombination mit } \\
\text { Tagdiensten }\end{array}$ & $\bullet$ & \\
\hline Wochenenddienste & $\bullet$ & \\
\hline Wochenend-Nachtdienste & $\bullet$ & \\
\hline
\end{tabular}

Quelle: Eigene Darstellung

\section{Planungshorizont und Flexibilität von Arbeitszeiten}

Bezüglich des Planungshorizontes sind einerseits die langfristige Vorhersehbarkeit des Dienstplans und andererseits die Häufigkeit kurzfristiger Änderungen relevant für die soziale Teilhabe. Die praktische Bedeutung einer Unterscheidung zwischen lang- und kurzfristiger Planung für die soziale Teilhabe zeigt sich besonders im Bereich der mobilen Pflege: 


\section{Planungshorizont: Mobile Pflege}

In der untersuchten Organisation der mobilen Pflege wird zwischen Dienstplanung (langfristig) und Einsatzplanung (kurzfristig) unterschieden. Die Beschäftigten schätzen die prinzipielle langfristige Planbarkeit der Arbeitszeiten in Form eines fixen 4-6-wöchigen Schichtrades sehr. Die kurzfristigen Änderungen durch den Arbeitgeber im Rahmen der Einsatzplanung oder sogar während laufender Dienste sind jedoch sehr belastend für die Planung der arbeitsfreien Zeit und soziale Teilhabemöglichkeiten.

Keine Garantie, weil wenn wer ausfällt, dann ist sowieso alles wieder anders, aber um einmal so grundsätzlich den Mitarbeitern eine gewisse Planbarkeit, auch der Freizeit, zu ermöglichen, dass man sagt: Okay, alle 4 Wochen, 6 Wochen wiederholt sich dieses Muster. (...) Aber wenn an einem Tag 4 Leute krankwerden, ist nix mehr mit Muster, ja? (mobile Pflege BL 1)

Für die Beschäftigten ist insbesondere herausfordernd, dass das tägliche Dienstende nur selten mit der geplanten Zeit übereinstimmt. Die Übernahme von KlientInnen erkrankter KollegInnen oder lange Wegstrecken mit schlechter Verkehrslage führen zu längeren Diensten:

Ja, also Termine einzuhalten ist für mich relativ schwierig im Privaten, weil wenn mich wer anruft und sagt: Wann hast du heute aus?, dann habe ich meinen Standardsatz: Wenn ich fertig bin, weil ich es nicht weiß. Es können Kunden wegfallen, es kann was dazukommen. Man kann um 14.00 Uhr noch einen Anruf kriegen: Kannst du noch der Kollegin helfen, die hat einen Arzttermin oder wie auch immer. Es gibt für uns eigentlich nicht wirklich irgendwelche Freizeiteinteilungen. (...) Also man kann da nicht wirklich sagen: Ich gehe heute einmal shoppen oder erledige einmal Weihnachtseinkäufe. Es kann dir wirklich dann passieren, dass du um $14.00 \mathrm{Uhr}$ einen Anruf kriegst: Du hast noch einen Ersteinsatz, der mitunter 90 Minuten dauert. Und schon ist der private Plan dahin. (mobile Pflege DGKP 3)

Auf diesem Weg aufgebaute Mehrstunden werden normalerweise bei der Erstellung des Dienstplans berücksichtigt und sukzessive stundenweise abgebaut. Die Beschäftigten würden jedoch einen geblockten Abbau in Form von ganzen Tagen bevorzugen. Gerade für die Gestaltung der Freizeit und den Erholungswert würde ihnen das subjektiv mehr bringen, als vereinzelt kürzere Arbeitstage. Selbstbestimmter Zeitausgleich ist auf bestimmte Wochentage begrenzt. De facto haben die verschiedenen Wochentage für viele Beschäftigte aus der Perspektive ihrer Nutzbarkeit für Freizeitaktivitäten und soziale Teilhabe aber unterschiedlichen Wert: „Ja, was mache ich an einem Dienstag?“ (Bereichsleitung mobile Pflege).

\section{Planungshorizont: Pflegeheim}

Im untersuchten Pflegeheim erfolgt die Bekanntgabe der Dienstpläne vier Wochen im Voraus. Selbstbestimmte Arbeitszeitflexibilität ist im Dienstplansystem insofern verankert, als die MitarbeiterInnen die Möglichkeit haben, pro Monat vier bis fünf Tage als wunschfrei zu markieren, 
was in den allermeisten Fällen auch berücksichtigt wird, dies wird im Zusammenhang mit sozialer Teilhabe positiv bewertet: „Wunschfrei hast du ein paar [Tage], die du angeben kannst. (...) Also 4 Tage hast du normal, 2 Wochenende und 2 Tage“ (Int. 2 Pflegeheim).

\section{Planungshorizont: Hotellerie}

Auch im Bereich der Nachtarbeit im Hotel zeigt sich die Bedeutung von guter Planbarkeit, vor allem was das Dienstende betrifft, für die Möglichkeiten sozialer Teilhabe. Im Beispiel der Nachtrezeption des untersuchten Hotels ist sich das Management der Bedeutung der Planungssicherheit für die Beschäftigten bewusst: „Planung. Genau. Und diese Dienstplansicherheit. Also planen kann ich viel, aber diese Sicherheit zu haben, dass der Plan dann auch hält" (Hotel BR).

Insbesondere ein fixes Dienstende ist wichtig, um die gewohnten Schlafenszeiten und davon abhängig den Plan der darauffolgenden Freizeit einhalten zu können. Ein großer Teil der MitarbeiterInnen im Hotel hat Migrationshintergrund. Es zeigt sich, dass diese Beschäftigten mitunter über spezifische Bedürfnisse sozialer Teilhabe verfügen. Zum Beispiel wird im untersuchten Hotel geschildert, dass Beschäftigte, um längere Aufenthalte bei Verwandten im Ausland organisieren zu können, Urlaub gerne geblockt konsumieren würden, idealerweise im Sommer. Diese Wünsche stehen dem Bedarf des Unternehmens entgegen, das in dieser Zeit üblicherweise sehr gut gebucht ist.

\section{Planungshorizont: Sicherheitsdienstleistung}

Von den untersuchten Organisationen ist die Sicherheitsdienstleistung jene mit dem größten Ausmaß an fremdbestimmter Flexibilität. Längerfristige Dienstpläne sind für einen großen Teil der Beschäftigten (circa 40\%) nicht üblich, da sie als Springer geführt werden und extrem flexible Arbeitseinsätze leisten. Auch für die restlichen $60 \%$ orientieren sich die Arbeitszeiten am Bedarf des Arbeitsgebers bzw. an den Anforderungen der KundInnen. Ein gewisses Maß an Mitbestimmung bei der Gestaltung der Dienstpläne ist gegeben, zugleich muss auch zu wenig attraktiven Zeiten wie am Wochenende oder an Feiertagen gearbeitet werden, d.h. hier wird das „notwendige Übel“ auf verschiedene Personen verteilt. Die hohen Anforderungen an die Beschäftigten in zeitlicher Hinsicht lassen sich vor allem deswegen durchsetzen, weil aufgrund der geringen Löhne in der Branche Zusatzdienste aus finanzieller Sicht für die Beschäftigten interessant sind.

40\% (...) sind Springerdienste. Das heißt, wenn wo ein Mitarbeiter ausfällt oder im Urlaub ist oder im Krankenstand (...) oder kurzfristig ein Zusatzauftrag reinkommt, dann werden diese Mitarbeiter aktiviert und werden teilweise tagesaktuell angerufen, ob sie nicht in den Dienst kommen können. Oder manche haben zwar einen Dienstplan, fällt ein Mitarbeiter aus, dann wird er Mittag angerufen an seinem freien Tag, ob er nicht Dienst machen könnte, weil wer ausgefallen ist. (Sicherheit BR 1)

Die Beschäftigten beschreiben durchaus Druck von Seiten des Arbeitgebers in Richtung der Übernahme zusätzlicher Dienste. Mitarbeiter, die kurzfristiges Einspringen mehrmals verweigern, sind im Unternehmen schlechter angesehen als solche, die sich zum Einspringen bereit 
erklären. Gerade kurzfristiges Einspringen an freien Tagen führt aber zu Problemen und belastet soziale Beziehungen stark. Diese Belastungen nimmt auch ein für die Diensteinteilung verantwortlicher Mitarbeiter wahr:

Und dann ist es natürlich irgendwann auch teilweise blöd, dass du dann vier Stunden vorher anrufst und sagst: Hey, kannst du bitte heute am Abend arbeiten? Drum sage ich, da gibt es dann schon viele, die in einer Beziehung sind, wo die Frau am Tag arbeitet, die natürlich sagen: So, jetzt weiß ich, ich habe 2 Tage frei, jetzt stelle ich mich sofort wieder auf Tag um, dass ich mit der Frau noch was machen kann. Und für die ist das natürlich, die sagen: Was soll ich dann? Jetzt stehe ich um 8.00 Uhr in der Früh auf und dann soll ich arbeiten gehen. (Sicherheit MA 1)

Ein wichtiger Aspekt ist daher der Ausgleich von Mehr- oder Überstunden in Form von Zeit und die Frage, wer über den Zeitpunkt, die Lage und das Ausmaß des Ausgleichs bestimmt. Zentral ist diese Frage, weil Freizeit abhängig von ihrer Lage auch über unterschiedlichen Wert verfügt.

\section{Planungshorizont: IT-Projektarbeit}

Das Kontrastbeispiel im Verhältnis von selbst- und fremdbestimmter Arbeitszeitflexibilität bildet die hochqualifizierte IT-Projektarbeit, wie sie beispielhaft in der Finanzdienstleistung und in Start-Up-Betrieben untersucht wurde. In letzteren verlangt die Arbeitsteilung viel Abstimmungsaufwand, weshalb u.a. Kernarbeitszeiten definiert sind. Darüber hinaus wird den MitarbeiterInnen viel Freiheit bei der Arbeitszeitgestaltung gewährt. Im Kontext von Selbst- vs. Fremdbestimmung ist die ausgeprägte Präferenz für Selbstbestimmung sowohl bei Unternehmensverantwortlichen als auch bei MitarbeiterInnen zu nennen. In der Praxis passt sich diese jedoch den unternehmerischen Bedürfnissen an. Dasselbe trifft auf die Beschäftigten bei der ITTochter eines Finanzdienstleisters zu.

Zwar finden wir dort eine präzise Vorausplanung und Abstimmung der betriebsnotwendigen Prozesse und eine ausgeprägte „Planungsdisziplin“, nichtsdestotrotz verfügen die Beschäftigten über große Freiheiten bei der kurzfristigen und langfristigen Planung ihrer Arbeitszeiten wie auch des Arbeitsortes. Planungs- bzw. Selbstorganisationskompetenz wird wie selbstverständlich vorausgesetzt, denn in diesem Unternehmen ist - wie überall dort, wo projektbasierte Arbeit vorrangig ist - indirekte Steuerung das entscheidende Kriterium gegenüber „bloßer“ Anwesenheit. Wie „großzügig“ die Interviewten mit ihren vorhandenen Zeitspielräumen umgehen, variiert je nach Beschaffenheit der Tätigkeit, privaten Verpflichtungen und wohl auch des Persönlichkeitstyps. Eine Business-Analytikerin schildert:

Es gibt bei mir schon viele Phasen, wie es momentan ist, dass ich deutlich Überstunden mache, also Mehrstunden, keine Überstunden. Und dann z.B., weil gerade schulfrei ist, mir Zeitausgleich nehme. (...) Es schwankt bei mir schon sehr, ja (lacht). Wenn ich meine Tochter in die Schule bringe, da bin ich um 8.30 da, aber Mittwoch und Donnerstag bringt sie mein Mann, da kann es auch sein, wenn ich daheim irgendetwas tun will, dass ich vielleicht erst um elf komme. (IT-Tochter MA 1) 


\section{Zusammenschau Planungshorizont und Flexibilität von Arbeitszeiten}

Wie im empirischen Material gut sichtbar wird, spielen für das Ausmaß und die Qualität der sozialen Teilhabe der Beschäftigten der Planungshorizont und die Flexibilität eine wichtige Rolle. Fragen, wie lange im Voraus bekannt ist, wann wie viel gearbeitet wird und mit welcher Verlässlichkeit diese Planung dann auch eintritt, sind zentral. Aus Sicht der Beschäftigten ist der Idealfall eine Kombination aus langfristiger verlässlicher Planung durch den Arbeitgeber und der Möglichkeit zu kurzfristiger selbstbestimmter Flexibilität.

Als besonders belastend für die soziale Teilhabe werden von den Befragten sehr kurzfristige fremdbestimmte Änderungen beschrieben. In diesen Fällen können langfristig geplante private Termine nicht mit Sicherheit zu- oder abgesagt werden. Private Entscheidungen müssen zeitnah getroffen werden und die Beschäftigten sind auf eine ebenso kurzfristige Planung ihres Umfeldes angewiesen. Als belastend wird auch beschrieben, wenn es zu kurzfristigem erzwungenem Zeitausgleich kommt, da dies zu ungeplanter Freizeit führt, die aus Sicht der Beschäftigten für soziale Teilhabe nicht gut genutzt werden kann. Insbesondere bei unregelmäßigen Diensten kann ein langer Planungshorizont die Belastungen entschärfen. Weiter erschwerend für die Möglichkeiten sozialer Teilhabe wirkt, wenn Einspringen kurzfristig bis spontan verlangt wird und es sich darüber hinaus um eine atypische Lage, insbesondere Nachtarbeit oder Wochenendarbeit handelt.

Als belastend für die soziale Teilhabe sind also kurzfristige Planung, geringe eigene Einflussmöglichkeiten und hohe kurzfristig fremdbestimmte Flexibilität festzustellen. Einen Überblick - basierend auf einer vergleichenden branchenübergreifenden Analyse - zur Wirkung der verschiedenen Faktoren im Zusammenhang mit Flexibilität und Planungshorizont von Arbeitszeit und sozialer Teilhabe gibt Tabelle 5.

Tabelle 5: Einfluss von Planungshorizont und Flexibilität der Arbeitszeit auf soziale Teilhabe

\begin{tabular}{|l|c|c|}
\hline $\begin{array}{l}\text { Planungshorizont und Flexi- } \\
\text { bilität von Arbeitszeit }\end{array}$ & $\begin{array}{c}\text { Belastend für soziale } \\
\text { Teilhabe }\end{array}$ & Hilfreich für soziale Teilhabe \\
\hline $\begin{array}{l}\text { Langfristige Dienstplanung } \\
\text { zum Beispiel in Form von } \\
\text { Schichträdern }\end{array}$ & & \\
\hline $\begin{array}{l}\text { Kurzfristige Dienstplanung } \\
\text { beispielsweise im 2-Wochen- } \\
\text { Rhythmus }\end{array}$ & $\bullet$ & \\
\hline $\begin{array}{l}\text { Möglichkeit zur langfristigen } \\
\text { Einplanung von Wünschen zur } \\
\text { Lage der Arbeitszeit }\end{array}$ & & \\
\hline $\begin{array}{l}\text { Hohes Ausmaß selbstbe- } \\
\text { stimmter kurzfristiger Flexibi- } \\
\text { lität }\end{array}$ & & \\
\hline $\begin{array}{l}\text { Hohes Ausmaß fremdbe- } \\
\text { stimmter kurzfristiger Flexibi- } \\
\text { lität }\end{array}$ & $\bullet$ & \\
\hline $\begin{array}{l}\text { Einspringen zu Zeiten mit aty- } \\
\text { pischer Lage }\end{array}$ & $\bullet$ & \\
\hline
\end{tabular}


Quelle: Eigene Darstellung

\section{Einschränkungen spezifischer Aspekte der sozialen Teilhabe am Beispiel der Pflege}

In den Interviews wurde auch erfragt, welche Aspekte der sozialen Teilhabe von Einschränkungen aufgrund der Arbeitszeit betroffen sind. Bestehen Freundschaften mit Personen, die im gleichen Beruf tätig sind, erleichtert dies die Koordination von Treffen. Dies hat jedoch auch mit dem Verständnis für die Arbeitssituation und nicht nur mit der tatsächlichen Verfügbarkeit zu tun. Kontakte zu Personen, die für Kinderbetreuung oder aufgrund von Pensionierung zu Hause sind, lassen sich leichter umsetzen:

Ist natürlich unter der Woche schon eher ein Bissl schwer, weil natürlich wir da - ich sage einmal - die meiste Zeit am Arbeiten sind. Aber es kommt auch immer draufan, was für einen Freundeskreis du hast. Ich habe einen mit vielen Mamas schon, die natürlich unter der Woche perfekt sind. (Pflegeheim Int 6)

Zu der Mama kann ich natürlich immer kommen. Die sitzt daheim immer und was weiß ich was alles. Aber sonst, wenn es jemand ist, der normale Arbeitszeiten hat, das überschneidet sich halt dann oft so. Das geht nicht gut. (Pflegeheim Int 4)

Die meisten von meinen Freundinnen arbeiten auch in der Pflege und haben auch viel unter der Woche frei. (...) Jetzt wollten wir gerade einen Termin finden für den Monat und haben genau einen Tag, wo wir alle miteinander frei haben. (lacht) (Pflege Int 7)

In Tabelle 6 wird am Beispiel von Beschäftigten in einem untersuchten Pflegeheim dargestellt, dass unregelmäßiger Wechseldienst mit Nacht- und Wochenendarbeit die stärksten Einschränkungen für Teilnahme an Vereinsaktivitäten, Kursen, politischen Aktivitäten usw. darstellt. Aktivitäten mit FreundInnen und Verwandten sind ebenfalls eingeschränkt. Wie sehr die Kernfamilie betroffen ist, hängt davon ab, welche Arbeitszeiten bei dem/der PartnerIn vorzufinden sind, und wie die Kinderbetreuung mit der Arbeit abgestimmt werden kann, das zeigen auch die folgenden beiden Zitate.

Mein Partner ist Koch. Der hat unter der Woche frei, z. B., und ich arbeite jetzt von Montag bis Freitag. (...) ja, ist natürlich schwierig zu handeln. Natürlich kann ich es mir jetzt im Laufe von der Dienstplangestaltung auch so einplanen, damit ich gemeinsam mit meinem Partner auch frei habe. (Pflegeheim Int 5)

Und mein Mann arbeitet auch eher am Wochenende, der ist selbständiger Fotograf und hat eigentlich am Wochenende immer Arbeit. [...] Von dem her ist das für mich kein Problem, wenn ich am Wochenende arbeite. (Pflegeheim Int 3) 
Tabelle 6: Beeinträchtigung von sozialer Teilhabe am Beispiel der Arbeit im Pflegeheim

\begin{tabular}{|l|l|l|}
\hline \multicolumn{2}{|l|}{$\begin{array}{l}\text { Arbeitszeitmodell: Unregelmäßiger Wechseldienst mit Nacht- und Wochen- } \\
\text { enddiensten }\end{array}$} \\
\hline \multirow{2}{*}{} & Kernfamilie: PartnerIn, Kinder & $\begin{array}{l}\text { Unterschiedlich: Hängt vom Arbeitszeitmuster } \\
\text { des Partners oder der Partnerin ab. Kinder: } \\
\text { Schwierigkeit, Kinderbetreuung und Dienste } \\
\text { abzustimmen. }\end{array}$ \\
\cline { 2 - 3 } & Familie: Verwandtschaft & $\begin{array}{l}\text { Einschränkung: Familienfeiern teilweise } \\
\text { schwierig mit dem Dienstplan zu vereinbaren. }\end{array}$ \\
\cline { 2 - 3 } & & $\begin{array}{l}\text { Einschränkung: Terminfindung kann aufwän- } \\
\text { dig sein, ist aber möglich. }\end{array}$ \\
& FreundInnen & $\begin{array}{l}\text { Hilfreich: FreundInnen aus gleicher Branche } \\
\text { mit Verständnis für arbeitszeitbedingte } \\
\text { Schwierigkeiten. }\end{array}$ \\
\cline { 2 - 3 } & & $\begin{array}{l}\text { Starke Einschränkung: Befragte geben fast } \\
\text { durchwegs Unmöglichkeit an. }\end{array}$ \\
\cline { 2 - 3 } & Verein, Kurs, &
\end{tabular}

Quelle: Eigene Darstellung

Anhand dieses Beispiels lässt sich gut nachvollziehen, dass unterschiedliche Arten der sozialen Interaktion außerhalb des Berufs unterschiedlich stark durch spezifische Arbeitszeitmodelle und auch Formen der Arbeitsorganisation betroffen sind. Es zeigt sich in den Interviews klar, dass regelmäßige Termine, zum Beispiel in Form von Vereinsarbeit, Stammtischen und Kursen bei Zeitkonflikten am stärksten gefährdet sind. Vergleichsweise hohe Priorität haben hingegen die Kontakte innerhalb der Kernfamilie. Abhängig von der Verteilung von Betreuungsaufgaben für Kinder innerhalb der Familien kommt ihnen bei einem Teil der Beschäftigten hohe oder höchste Priorität zu.

\section{Diskussion}

Im Zentrum des vorliegenden Artikels stand die Frage, wie sich atypische Arbeitszeiten auf die Möglichkeiten der Beschäftigten zu sozialer Teilhabe im Sinne sozialer Interaktion außerhalb des Arbeitszusammenhangs auswirken. Es lässt sich festhalten, dass die untersuchten Arbeitszeitmerkmale Dauer, Lage und Planungshorizont sowohl sozial belastende als auch entlastende Wirkungen entfalten können.

Als belastend wurden insbesondere folgende Merkmale ermittelt:

- Viele lange Dienste

- Kurze / zu wenig Erholungsphasen zwischen Diensten

- Atypische Lage der Arbeitszeit (frühe / späte Dienste, wechselnde Randdienste, Nachtarbeit, Wochenendarbeit)

- Kurzfristige Planung / wenig langfristige Vorhersehbarkeit

- Spontane fremdbestimmte Änderung der bestehenden Dienstpläne 
Insgesamt führt die Kombination aus Nachtarbeit, wechselnden Diensten und kurzfristigen Arbeitseinsätzen, die vor allem in der Sicherheitsbranche zu finden ist, zur höchsten hier beobachteten Belastung in Hinblick auf die soziale Teilhabe der GesprächspartnerInnen. Über unbedingt notwendige Erholung, Hausarbeiten, Kinderbetreuung und die Pflege des Familienlebens hinaus bleibt in diesen Fällen kaum mehr Zeit für Aktivitäten.

Die hier vorgestellten qualitativen, explorativen Ergebnisse bestätigen und ergänzen somit bisherige Befunde zu den sozialen Effekten der Arbeitszeitgestaltung (Arlinghaus et al. 2019; Wöhrmann et al. 2020). Eine Erweiterung bisheriger Erkenntnisse ist hier der Aspekt des frühen Arbeitsbeginns, der bereits am Vorabend wirkt, sowie die Kombinationswirkung verschiedener Belastungsfaktoren.

Die vorliegende Studie trägt zudem dazu bei, das Zusammenwirken be- und entlastender Mechanismen der Arbeitszeit besser zu verstehen. Als entlastende oder für die soziale Teilhabe förderliche Arbeitszeitmerkmale wurden folgende Aspekte ermittelt:

- Lange Freizeitphasen

- Zeitbonus für belastende Arbeitszeiten

- Reduzierte Arbeitsstunden

- Langfristige Dienstplanung

- Eigener Einfluss auf die Arbeitszeit, z.B. Wünsche einplanen

- Kurzfristige selbstbestimmte Flexibilität

Diese entlastenden Merkmale können die Gesamtbelastung aus Sicht der Befragten reduzieren. Dies wird insbesondere bei langen täglichen Arbeitszeiten sichtbar, die von den Beschäftigten aufgrund der oft mit ihnen verbundenen langen Freizeitphasen aus sozialer Sicht durchaus auch positiv bewertet werden. Diese subjektiv als gut erlebte Passung zu den eigenen Lebensumständen schützt aber nicht vor negativen gesundheitlichen Folgen, wenn ausreichende Ruhezeiten nicht eingehalten werden können (vgl. exemplarisch: Blasche et al. 2017). Diese Ergebnisse stehen ebenfalls im Einklang mit bestehenden quantitativen Erkenntnissen (z.B. Arlinghaus et al. 2019), wobei in bisherigen Studien nach unserem Wissensstand selten eine Kombination dieser Merkmale untersucht wurde.

Beschäftigte und deren Angehörige stehen vor der Herausforderung, ihr Leben mit dem ihres Umfelds so gut wie möglich zu synchronisieren. In der österreichischen Gesellschaft orientiert sich der soziale Rhythmus grundsätzlich an ca. 8 Stunden Arbeit tagsüber mit einer anschließenden Freizeitperiode. Die westliche Gesellschaft ist zudem eine Abend- und Wochenendgesellschaft wodurch in hohem Maße normativ vorgegeben ist, welche Zeiten sozial besonders wertvoll sind. Die hier dargestellten Ergebnisse bestätigen Studien (z.B. Arlinghaus et al. 2019) zu sozial wirksamer Freizeit, die zeigen, dass zum Beispiel Deutschland über die letzten 30 Jahre unverändert als Abend- und Wochenendgesellschaft (im Gegensatz zu einer Rundum-die-Uhr-Gesellschaft) beschrieben werden kann. Daher beschränken bestimmte Arbeitszeitmuster (insbesondere bei Abend- und Wochenendarbeit) die soziale Nutzbarkeit und erschweren die Synchronisation des Soziallebens mehr als andere.

Selbstbestimmte Flexibilität bietet hingegen deutliche Vorteile für eine gute Work-Life Balance, wie in unseren Ergebnissen sichtbar wird. Eine langfristige Planbarkeit und selbstbestimmte Flexibilitätsformen erleichtern die soziale Teilhabe, wohingegen kurzfristiges Einspringen oder unternehmensseitige Flexibilität als hinderlich wahrgenommen werden. Auch 
dieses Ergebnis ist in Einklang mit bisherigen Befunden (Arlinghaus et al. 2019). Über bisherige Studien hinaus wurde zudem hier untersucht, welche einzelnen Aspekte der sozialen Teilhabe besonders durch die Arbeitszeitgestaltung betroffen sind. Das System der sozialen Interaktion besteht außerhalb der Erwerbsarbeit aus Partner / Partnerin, Kindern, sonstigen Angehörigen, FreundInnen, Bekannten, NachbarInnen, KollegInnen in Vereinen, etc. Je nachdem, worauf der Verweis auf soziale Teilhabe abzielt bzw. welche Handlungsbezüge oder Kontaktgruppen durch lange oder atypische Erwerbsarbeitszeiten beeinträchtigt sind, ergeben sich zum Teil unterschiedliche Implikationen. Schränkt die Arbeitszeit die soziale Teilhabe ein, zeigen sich in unserer Studie Prioritätensetzungen im Rahmen der individuellen Möglichkeiten, die Form eines Schichtenmodells dargestellt werden können (siehe Abbildung 1). Unter zunehmend erschwerten Bedingungen der Synchronisation scheint es zu einer schrittweisen Einschränkung der unterschiedlichen Arten von sozialer Teilhabe von außen nach innen zu kommen.

\section{Abbildung 1: Schichtenmodell zur Beeinträchtigung sozialer Teilhabe}

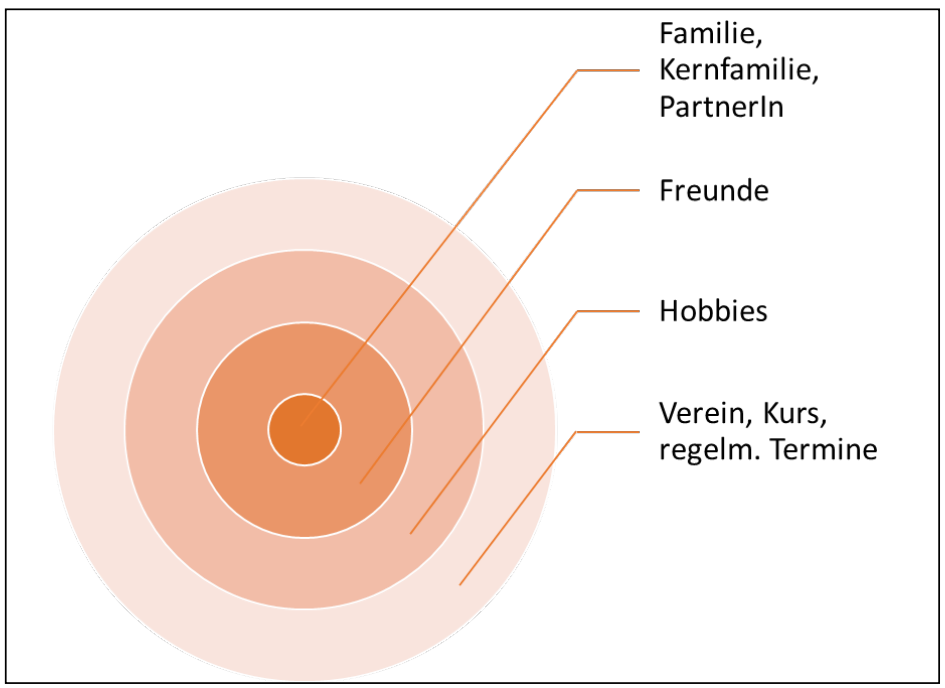

Quelle: Eichmann et al. (2019)

Familienzeit scheint also auf den ersten Blick jene Zeitverwendungskategorie zu sein, die durch ausufernde oder atypische Erwerbsarbeitszeiten bzw. allgemein durch Zeitnot am ehesten Leidensdruck erzeugt. Dies jedoch - so zeigt die vorliegende Empirie - insbesondere deswegen, weil sie als jener Zeitraum zu verstehen ist, auf den sich die sozialen Kontakte reduzieren, wenn es darum geht, Prioritäten setzen zu müssen. Besteht die Gefahr, dass auch diese Zeitressourcen zur Disposition stehen, spitzt sich die Situation in der Form zu, dass kaum mehr andere außerberufliche Sozialkontakte möglich sind.

Während Stärken der präsentierten Studie in dem qualitativen Vorgehen liegen, das eine große Tiefe der Untersuchung ermöglicht und die Arbeitszeitbedingungen in spezifischen Branchen aufgreift, sind einige Limitationen zu beachten. Zum einen handelt es sich nicht um eine repräsentative Studie der untersuchten Branchen, wodurch die Ergebnisse nicht zwangsläufig auf alle Beschäftigten in diesen Branchen generalisiert werden können. Das qualitative und explorative Vorgehen ermöglicht zudem keine statistische Prüfung von Unterschieden oder die Ermittlung bestimmter Effektstärken. Die ermittelten möglichen Zusammenhänge 
sollten daher anhand konkreter Hypothesen in weitergehenden qualitativen und umfangreicheren Stichproben, wenn möglich prospektiv oder als Interventionsstudie, getestet werden.

\section{Fazit}

In politischen Debatten geht es bei der Frage nach der Gestaltung von Arbeitszeiten und ihrer Vereinbarkeit mit Zeit für außerberufliche Beziehungen vorwiegend um die Zeit mit der Familie. Zusätzlich steht die Frage der Dauer im Zentrum dieser Debatten. Je länger gearbeitet wird, umso schlechter ist dies für die Vereinbarkeit von Arbeit und Familie. Es zeigt sich jedoch, dass dieser begründete und fraglos richtige Befund, zu kurz greift, da Beeinträchtigungen anderer gesellschaftlich und individuell relevanter Lebensbereiche ausgeklammert werden. Ein wichtiges Thema in diesem Kontext ist die Mitgliedschaft in Vereinen, die Teilnahme an Kursen und das Einhalten von regelmäßigen Terminen insgesamt. Weiter gedacht leidet die politische Teilhabe im Sinne einer demokratischen Partizipation in der Zivilgesellschaft insgesamt, beginnend mit der ehrenamtlichen Mitarbeit in Interessenvertretungen, Vereinen oder der informellen Freiwilligenarbeit und Nachbarschaftshilfe bis hin zur Teilnahme an Protestformen wie Demonstrationen und anderen politischen Aktivitäten.

Um die Chance auf zufriedenstellende soziale Teilhabe zu verbessern, erweisen sich aus Beschäftigtenperspektive unterschiedliche Aspekte als bedeutend. In erster Linie geht es um langfristige Planbarkeit der Arbeitszeiten mit ausreichend Mitgestaltungsmöglichkeiten durch die ArbeitnehmerInnen. Darauf aufbauend zeigt sich, dass die Verlässlichkeit dieser Planung von großer Relevanz ist, d.h. dass es nur zu geringen und seltenen kurzfristigen Änderungen durch den Arbeitgeber kommt. In den meisten hier dargestellten Fällen scheitert dies an einer zu dünnen Personaldecke. Selbstbestimmte zeitliche Flexibilität ist für die Beschäftigten von großem Vorteil, zumindest so lange sie nicht durch die Flexibilität der KollegInnen in der eigenen Planungssicherheit eingeschränkt werden.

\section{Danksagung}

Die AutorInnen bedanken sich bei den InterviewpartnerInnen für Ihre Offenheit, ihre persönliche Wahrnehmung zu teilen. Dank gilt darüber hinaus den beiden anonymen GutachterInnen und den RedaktorInnen des Journals sozialpolitik.ch für die wertvollen Hinweise und Kommentare.

\section{Deklaration von Interessenkonflikten}

Die AutorInnen deklarieren keine Interessenkonflikte in Bezug auf Forschung, AutorInnenschaft und/oder Publikation des Artikels. 


\section{Finanzierung}

Dieser Text basiert auf den Ergebnissen eines durch die Österreichische Forschungsförderungsgesellschaft (FFG) geförderten Projekts mit dem Titel Soziale Teilhabe als Herausforderung für Arbeitszeitgestaltung - Indikatoren-Entwicklung für faire Bewertungssysteme betrieblicher Gestaltung von Arbeitszeit, das zwischen Januar 2018 und Oktober 2019 in Österreich durchgeführt wurde.

\section{Literaturverzeichnis}

Amlinger-Chatterjee, Monisha (2016). Psychische Gesundheit in der Arbeitswelt - Atypische Arbeitszeiten. Dortmund: Bundesanstalt für Arbeitsschutz und Arbeitsmedizin.

Arlinghaus, Anna, Johannes Gärtner, Sylvia Rabstein, Sebastian Schief und Céline Vetter (2016). Dauernachtarbeit - Eine Sichtung des vorhandenen Wissenstandes mit Thesen, Empfehlungen \& Forschungsfragen. Sozialpolitik.ch, 2/2016, 1-11. Zugriff am 3.8.2020 auf:

http://dx.doi.org/10.18753/2297-8224-76

Arlinghaus, Anna, Philip Bohle, Irena Iskra-Golec, Nicole Jansen, Sarah Jay und Lucia Rotenberg (2019). Working Time Society consensus statements: Evidence-based effects of shift work and non-standard working hours on workers, family and community. Industrial Health, 57, 184-200. Zugriff am 3.8.2020 auf: https://www.jstage.jst.go.jp/article/indhealth/57/2/57 SW-4/ pdf.

Astleithner, Franz und Bettina Stadler (2018). Flexible Arbeitszeitarrangements aus der Perspektive österreichischer ArbeitnehmerInnen. Wien: AK Wien.

Blasche, Gerhard, Verena-Maria Bauböck und Daniela Haluza (2017). Work-related self-assessed fatigue and recovery among nurses. International Archives of Occupational and Environmental Health, 90(2), 197-205. Zugriff am 3.8.2020 auf:

https://link.springer.com/content/pdf/10.1007/s00420-016-1187-6.pdf.

Eichmann, Hubert, Bernhard Saupe, Annika Schönauer, Bettina Stadler, Anna Arlinghaus und Johannes Gärtner (2019). Soziale Teilhabe als Herausforderung für Arbeitszeitgestaltung. Indikatoren-Entwicklung für faire Bewertungssysteme betrieblicher Gestaltung von Arbeitszeit. Projektbericht, FORBA, XIMES, Wien. Zugriff am 3.8.2020 auf:

https://www.forba.at/en/bericht/soziale-teilhabe-als-herausforderung-fuer-arbeitszeitgestaltungindikatoren-entwicklung-fuer-faire-bewertungssysteme-betrieblicher-gestaltung-von-arbeitszeit/.

Hochschild, Arlie Russel (2006). Keine Zeit. Wenn die Firma zum Zuhause wird und zu Hause nur Arbeit wartet (2. Auflage). Wiesbaden: VS Verlag.

Kümmerling, Angelika (2007). Arbeiten, wenn andere frei haben: Nacht- und Wochenendarbeit im europäischen Vergleich. Duisburg: Universität Duisburg-Essen.

Marschke, Britta und Heinz Ulrich Brinkmann (Hg.) (2011). Handbuch Migrationsarbeit. Wiesbaden: VS Verlag.

Mayring, Philipp und Thomas Fenzl (2019). Qualitative Inhaltsanalyse. In: Baur, Nina und Jörg Blasius (Hg.) Handbuch Methoden der empirischen Sozialforschung. Wiesbaden: Springer VS, 543-556. 
Pomberger, Markus (2005). Wie neuartig sind flexible Arbeitszeiten? Historische Grundlinien der Arbeitszeitpolitik. In: Seifert, Hartmut (Hg.). Flexible Zeiten in der Arbeitswelt. Frankfurt: Campus Verlag, 9-39.

Simmel, Georg (2013 [1908]). Soziologie. Untersuchungen über die Formen der Vergesellschaftung (7. Auflage). Berling: Duncker \& Humblot.

Seifert, Hartmut (Hg.) (2005). Flexible Zeiten in der Arbeitswelt. Frankfurt: Campus Verlag.

Tönnies, Ferdinand (1920 [1887]). Gemeinschaft und Gesellschaft: Grundbegriffe der reinen Soziologie (3. Auflage). Berlin: Wissenschaftliche Buchgesellschaft.

Tucker, Philip (2006). Compressed working weeks. Geneva: International Labor Office.

Weber, Max (2002 [1922]). Wirtschaft und Gesellschaft (5. Auflage). Tübingen: Mohr Siebeck.

Wirtz, Anna (2010). Gesundheitliche und soziale Auswirkungen langer Arbeitszeiten. Dortmund: Bundesanstalt für Arbeitsschutz und Arbeitsmedizin. Zugriff am 3.8.2020 auf: https://www.baua.de/DE/Angebote/Publikationen/Berichte/Gd59.pdf.

Wöhrmann, Anne, Grit Müller und Kathrin Ewert (2020). Shift Work and Work-Family Conflict: A Systematic Review, Sozialpolitik.ch (dieses Heft). 